\title{
BMJ Open A systematic review to identify and assess the effectiveness of alternatives for people over the age of 65 who are at risk of potentially avoidable hospital admission
}

\author{
Alyson L Huntley, ${ }^{1}$ Melanie Chalder, ${ }^{1,2}$ Ali R G Shaw, ${ }^{1}$ William Hollingworth, ${ }^{3}$ \\ Chris Metcalfe,,$^{1,4}$ Jonathan Richard Benger, ${ }^{5,6}$ Sarah Purdy ${ }^{1}$
}

To cite: Huntley AL, Chalder M, Shaw ARG, et al. A systematic review to identify and assess the effectiveness of alternatives for people over the age of 65 who are at risk of potentially avoidable hospital admission. BMJ Open 2017;7:e016236. doi:10.1136/ bmjopen-2017-016236

- Prepublication history and additional material are available. To view these files please visit the journal online (http://dx.doi org/10.1136/bmjopen-2017016236).

Received 2 February 2017

Revised 31 May 2017

Accepted 31 May 2017

\section{(a) CrossMark}

For numbered affiliations see end of article.

Correspondence to

Dr Alyson L Huntley;

alyson.huntley@bristol.ac.uk

\section{ABSTRACT}

Background/objectives There are some older patients who are 'at the decision margin' of admission. This systematic review sought to explore this issue with the following objective: what admission alternatives are there for older patients and are they safe, effective and cost-effective? A secondary objective was to identify the characteristics of those older patients for whom the decision to admit to hospital may be unclear.

Design Systematic review of controlled studies (April 2005-December 2016) with searches in Medline, Embase, Cinahl and CENTRAL databases. The protocol is registered at PROSPERO (CRD42015020371). Studies were assessed using Cochrane risk of bias criteria, and relevant reviews were assessed with the AMSTAR tool. The results are presented narratively and discussed.

Setting Primary and secondary healthcare interface. Participants People aged over 65 years at risk of an unplanned admission.

Interventions Any community-based intervention offered as an alternative to admission to an acute hospital. Primary and secondary outcomes measures Reduction in secondary care use, patient-related outcomes, safety and costs.

Results Nineteen studies and seven systematic reviews were identified. These recruited patients with both specific conditions and mixed chronic and acute conditions.

The interventions involved paramedic/emergency care practitioners $(n=3)$, emergency department-based interventions $(n=3)$, community hospitals $(n=2)$ and hospital-at-home services $(n=11)$. Data suggest that alternatives to admission appear safe with potential to reduce secondary care use and length of time receiving care. There is a lack of patient-related outcomes and cost data. The important features of older patients for whom the decision to admit is uncertain are: age over 75 years, comorbidities/multi-morbidities, dementia, home situation, social support and individual coping abilities.

Conclusions This systematic review describes and assesses evidence on alternatives to acute care for older patients and shows that many of the options available are safe and appear to reduce resource use. However, cost analyses and patient preference data are lacking.

\section{Strengths and limitations of this study}

- High-quality systematic review of controlled studies.

- Specific focus on admission avoidance interventions for acute care of older people.

- Studies cover a wide range of acute conditions and acute exacerbation of chronic conditions in older people.

- Some of the studies are pragmatic in approach and are at high risk of bias.

- Most studies do not provide associated costs/cost analyses of interventions or patient preference data.

\section{INTRODUCTION}

Reducing emergency bed days is one of the biggest challenges currently facing the National Health Service (NHS) in the UK, and there is considerable pressure to reduce hospital admissions among older people throughout the developed world. ${ }^{1}$ It has been suggested that clinicians should "choose to admit only those frail older people who have evidence of underlying life-threatening illness or need for surgery. ${ }^{2}$ In the UK, there has been a $65 \%$ increase in hospital admissions for those over 75 years of age in the last decade. Furthermore, people over 85 years of age now account for $11 \%$ of emergency admissions and $25 \%$ of critical care bed days. ${ }^{3}$ The international literature indicates that decisions to admit to an acute hospital (AH) are often influenced by inadequate knowledge of the patient or condition, communication difficulties between primary and secondary care, presence of comorbidities, availability of test results, perceived benefits of in-patient care and patient preferences. ${ }^{4} \mathrm{~A}$ review by NHS England highlighted the need to identify those frail and elderly people who need care but do not have a medical need requiring 
hospital admission. ${ }^{3}$ It is clear that there are some older patients for whom care in the community is safe, perhaps with provision of additional services, and some for whom admission is required to deliver diagnostics or treatment that are only available in a hospital. However, for those patients 'at the decision margin,' the best path of action may be unclear. ${ }^{5}$ The decision may be affected by non-clinical and clinical factors (eg, multi-morbidity, how much risk the patient or family is willing to accept).

Our specific objective was to conduct a systematic review to identify studies of community-based interventions aimed at reducing secondary care use in older patients with acute medical problems potentially requiring unscheduled hospital admission. A secondary objective was to further confirm the characteristics of those older patients for whom the decision to admit to hospital may be unclear.

\section{METHODS}

\section{Protocol and registration}

The protocol for the systematic review was registered at the PROSPERO register on 14June 2015. The registration number is CRD42015020371 (supplementary material).

\section{Eligibility criteria}

Publications of any randomised or non-randomised controlled trial (RCT or nRCT) that fitted our PICO criteria: a Population aged over 65 years, of either sex living in Organisation for Economic Co-operation and Development countries being considered for an unplanned admission, receiving either an Intervention considered to be an alternative to $\mathrm{AH}$ admission or $\mathrm{AH}$ admission (Control). The studies needed to record at least one of the following as either a primary or secondary Outcome: intervention effectiveness in terms of patient's subsequent emergency department (ED) attendance or readmission, patient-related outcomes, safety or healthcare costs.

\section{Information sources and searches}

Medline, Medline In-Process, Embase, Cinahl and CENTRAL databases were searched from January 2005 to April 2015 inclusively using search terms based on the eligibility criteria (online supplementary appendix 1). An update was run in December 2016 across Medline and Medline In-Process. We included any relevant systematic reviews published 2010-2016. The decision to time limit the searches was based on the fact that the systematic reviews would cover any older studies and that any evidence not included in these two sources was unlikely to be relevant to the fast-changing primary and secondary healthcare interface. The King's Fund and Agency for Healthcare Research and Quality websites were also searched in April 2015. ${ }^{6} 7$ References were managed using EndNote X6 software and were screened by title and abstract followed by full text, both independently and in duplicate (AH, BD), using predefined inclusion/ exclusion criteria. Any disagreements in either stage were resolved using a third reviewer (SP). The reference lists of included studies were checked, and forward referencing was conducted using Google Scholar. Authors of included studies were contacted for details of any extra studies.

\section{Data items and collection process}

Data from all primary studies (2005-2016) were extracted into a custom-designed table. The main results and conclusions of recent high-quality systematic reviews (2010-2016) that included relevant primary studies were also recorded.

\section{Assessment of risk of bias of individual studies}

The Effective Practice and Organisation of Care Cochrane risk of bias tool was used to critically appraise RCTs and nRCTs (online supplementary appendix 2). ${ }^{8}$

\section{Assessment of methodological quality of systematic reviews (AMSTAR)}

The AMSTAR checklist was used to assess the quality of the included systematic reviews (online supplementary appendix 3). ${ }^{9}$

\section{Synthesis of results}

The data are presented narratively describing, if present, the most relevant systematic review and/or individual studies for each intervention and, where appropriate, for a specific condition.

In order to identify the characteristics of those older patients for whom the decision to admit to hospital may be unclear, the inclusion/exclusion criteria and demographics of the participants were examined and key features were tabulated alongside the number and references of relevant studies.

\section{RESULTS}

The systematic review identified four types of intervention from across 19 studies published in 24 papers: paramedic practitioners (PPs)/emergency care practitioners (ECPs) $(n=3)$, ED interventions $(n=3)$, community hospitals $(\mathrm{CHs})(\mathrm{n}=2)$ and hospital-at-home $(\mathrm{HaH})$ services $(\mathrm{n}=11)^{10-33}$ (PRISMA diagram) (online supplementary file 4). Ten of the included studies were RCTs and nine were nRCTs (online supplementary summary table). Fifteen studies were conducted in western European countries of which four were in the UK. Two studies were conducted in Australia and two studies in the USA. Risk of bias, general intervention description, AMSTAR and study data are detailed in the appendices (online supplementary appendices $1-4$, supplementary file 6 ). There was an obvious divide between risk of bias of RCTs and nRCTs with the RCTs generally at low risk for most domains, although for some domains, there was insufficient information to be make a judgement (online supplementary appendix 2). The nRCTs were at high risk from not being randomised, and in some studies, there was a suggestion of health professional choice in allocation and, as with the RCTs, information was sometimes lacking. Risk of 
bias of individual studies is detailed below in the relevant section.

The AMSTAR ratings of the systematic reviews were generally good, although some reviews did not list details of excluded studies, included studies of high risk of bias and did not perform publication bias analysis (online supplementary appendix 3 ).

\section{PP/ECP interventions}

Three studies were identified ${ }^{10-12}$ and no relevant recent systematic reviews (online supplementary appendix 4).

A cluster RCT compared PPs with additional training $(\mathrm{n}=1469)$ with standard PPs $(\mathrm{n}=1549)$ in assessing and treating elderly people following 999 calls with the aim of measuring subsequent emergency care. ${ }^{10}$ Similarly, two more recent nRCT investigated the role of ECPs in avoiding ED attendance/admissions in elderly populations. ${ }^{11} 12$ Gray performed a case-series study of ECP attendances for elderly patients aged over 65 years with a fall $(n=233)$ compared with historical controls $(n=772)$, and Mason et al performed a cluster controlled study of enhanced ECP care for five care homes $(n=256)$ compared with standard care in five other care homes $(n=201)$. Risk of bias was low for all the domains of the cluster RCT, and both of the nRCTs were at high risk due to lack of randomisation.

In the cluster RCT, all primary outcomes comparing the intervention with the control group were improved: relative risk of ED attendance within 28 days (RR 0.72 $(0.68,0.75))$, relative risk of hospital admission within 28 days (RR $0.87(0.81,0.94))$, being very satisfied with care (RR $1.16(1.09,1.23))$ and mean total episode duration in hours $(-42.2(-59.5,-25.0))$ with a reported $\mathrm{p}<0.001$ for all. ${ }^{10}$ The secondary outcome of mortality was comparable between groups, but intervention patients had a greater number of subsequent unplanned contacts with secondary care at 28 days ( 330 vs $259, \mathrm{p}<0.01)$.

The two nRCTs reported a greater reduction in admissions when comparing the intervention with normal ECP practice, but these results are of limited use due to the high risk of bias of the studies. ${ }^{11} 12$

None of the studies of PP/ECP interventions provided details of cost data or cost-effectiveness analysis.

\section{ED interventions}

The searches identified one RCT (Sun et al) that was assessed to be at low risk of bias, and two nRCTs (Benaiges $e t a l$, Salvi $e t a l$ in which the risk of bias was high for several domains including randomisation. ${ }^{13-15}$ No relevant, recent systematic reviews were identified (online supplementary appendix 4).

Sun and colleagues conducted an RCT in which patients attending ED with syncope were randomised to receive either a syncope protocol in an observation unit $(\mathrm{n}=62)$ or usual care $(\mathrm{n}=62)^{13}$ where the maximum stay in the observation unit could not exceed than 24 hours.

In terms of primary outcomes, patients randomised to the intervention spent less time in hospital at the index visit (29 vs 47 hours $\mathrm{p}<0.001$ ) and were less likely to be admitted to hospital (RR 0.16 (95\% CI 0.09 to 0.29 ), $\mathrm{p}<0.001)$. There were no differences in the secondary outcomes of serious events, quality of life (QoL) or satisfaction with care between groups. A reduction in costs was reported, but no formal statistical comparison was performed (index visit: US $\$ 1400$ vs US $\$ 2420 ; 30$ days: US $\$ 1800$ vs US $\$ 2520$ (2011 data)).

The first of the two nRCT compared usual care with treatment in a 'day hospital' for hyperglycaemic crisis from which the main result was improved readmission rates and associated costs (Benaiges et al), while the second nRCT compared a specialist geriatric ED intervention with a standard ED procedure (Salvi et al) but without evidence of any differences in outcome and had significant differences in baseline demographic data. ${ }^{14} 15$

\section{CH interventions}

Two RCTs were identified describing a $\mathrm{CH}$ intervention as an alternative to $\mathrm{AH}$ care ${ }^{16-19}$ and no relevant, recent systematic reviews (online supplementary appendix 4).

Both RCTs were at low risk of bias overall. In the RCT by Vicente $e t$ al, participants were randomised following triage at home to either go to $\mathrm{CH}(\mathrm{n}=410)$ or to ED $(\mathrm{n}=396) .{ }^{16}$ The data presented were limited. The authors reported that the nurse attending the patient at home sent 90 intervention participants to $\mathrm{CH}$ (primary outcome), although 6 of those individuals were subsequently transferred from $\mathrm{CH}$ to ED (secondary outcome). There were no formal statistical analyses, and cost data were not presented.

The Garåsen RCT compared $\mathrm{CH}$ care $(\mathrm{n}=72)$ with $\mathrm{AH}$ care $(n=72)$ and was published over three separate papers. ${ }^{17-19}$ There was no distinction between primary and secondary outcomes. At 26 weeks, there were fewer readmissions in the $\mathrm{CH}$ group versus the $\mathrm{AH}$ group (19\% vs $36 \%, p=0.02)$ and more people receiving no care $(25 \%$ vs $10 \%, \mathrm{p}=0.01$ ). At 12 months, there were fewer deaths in the $\mathrm{CH}$ group ( $18 \%$ vs $31 \%, \mathrm{p}=0.03$ ), although the observation period was considerably longer in the $\mathrm{CH}$ group (335.7 vs 292.8 days, $\mathrm{p}=0.01$ ). Total cost of treatment was less in the $\mathrm{CH}$ group compared with those receiving AH care: NOK 39650 ((95\% CI NOK 30996 to 48304) vs NOK 73417 (95\% CI NOK 52992 to 93843)), data collected in 2003-2005 ( $\mathrm{p}=0.002)$. Average health services costs per patient/day for the entire observation period was NOK 606 (95\% CI NOK 450 to 761) in the $\mathrm{CH}$ group compared with NOK 802 (95\% CI NOK 641 to 962) in the AH group ( $\mathrm{p}=0.026)$.

\section{HaH interventions}

Eight of the HaH studies were focused on specific conditions: heart failure $(\mathrm{HF}) \quad(\mathrm{n}=3)$, chronic obstructive pulmonary disease (COPD) $(\mathrm{n}=1)$, pulmonary embolism $(\mathrm{n}=1)$, pneumonia $(\mathrm{n}=1)$, stroke $(\mathrm{n}=1)$ and uncomplicated diverticulitis $(n=1) .^{20-28}$ The remaining three $\mathrm{HaH}$ studies recruited older participants with a range of conditions, and two of these recruited from residential homes. ${ }^{29-33}$ All the specific condition studies were included in recent (2010-2016) systematic reviews, ${ }^{34-40}$ 
but no relevant reviews for the older participants with a range of conditions were identified (online supplementary appendix 4$)$.

\section{Heart failure}

Three RCTs were identified on $\mathrm{HaH}$ for HF, and their results were published in four separate papers. ${ }^{20-23}$ These studies were included in two previous reviews of $\mathrm{HaH}$, one of which focused on HF (Quaddoura et al) ${ }^{34}{ }^{35}$ This review used the Cochrane risk of bias tool and described the overall quality of the RCTs as modest. The AMSTAR rating of the review highlighted a lack of description of excluded studies and the combination of different QoL measures in meta-analysis.

In the Quaddoura systematic review, the patients were randomised to either $\mathrm{HaH}$ or $\mathrm{AH}$ within the $\mathrm{ED}$ and the primary outcomes of the review were hospital readmissions and mortality. $\mathrm{HaH}$ increased time to first readmission (mean difference 14.13 days $(95 \%$ CI 10.36 to 17.91), $\mathrm{p}=0.015$ ) using data from two RCTs $(\mathrm{n}=132),{ }^{22}{ }^{23}$ although there was no strong evidence of an effect on the rate of readmission (RR $0.68(0.42,1.09)$ ) using data from two RCTs $(\mathrm{n}=172) .{ }^{20}{ }^{22}$ This is a sizeable reduction but consistent with chance in a data set of this size. An improvement was reported in health-related QoL at both 6 and 12 months (standardised mean difference (SMD) $-0.31(-0.45$ to -0.18$)$; SMD -0.17 ( -0.31 to $-0.02)$, respectively). $\mathrm{HaH}$ was comparable to $\mathrm{AH}$ care on all-cause mortality $(\operatorname{RR} 0.94(0.67,1.32))$ using data from all three RCTs. These studies also showed a significant reduction in costs for the index treatment period $(p<0.001)$. Two trials ${ }^{20}{ }^{23}$ reported lower costs in the $\mathrm{HaH}$ group at 12 months, although the difference was not statistically significant in one of the studies. ${ }^{20}$ When the authors of this particular review calculated total costs for these two trials, both indicated a cost reduction for $\mathrm{HaH}$ compared with AH care.

\section{Chronic obstructive pulmonary disease}

An RCT by Ricauda was published in 2008 and was also included in two recent systematic reviews, one focusing on COPD and one more generally on HaH. ${ }^{24} 3536$ The high-quality COPD review included eight RCTs, one of which described $\mathrm{HaH}$ in an early discharge setting, plus the Ricauda trial and six that were published prior to our 2005 inclusion date.

The Ricauda RCT compared HaH $(n=52)$ with AH $(n=52)$ and was conducted with low risk of bias. The primary outcomes were hospital readmission and mortality rates at 6 months. The secondary outcomes included a range of depression, functional, cognitive and nutritional measures as well as costs.

The study showed that there were fewer hospital readmissions for $\mathrm{HaH}$ patients compared with $\mathrm{AH}$ patients at 6 months ( $42 \%$ vs $87 \%$, $\mathrm{p}=0.001$ ), although $\mathrm{HaH}$ patients had a longer length of stay than those in the AH group (15.5 $\mathrm{SD} \pm 9.5$ vs $11.0 \pm \mathrm{SD} 7.9$ days, $\mathrm{p}=0.01$ ). While $\mathrm{HaH}$ patients experienced improvements in depression and QoL scores during the study, there was no evidence of difference between the two groups for these outcomes at 6 months. Cumulative mortality at 6 months was comparable between groups $(20.2 \%)$.

All patients discharged from $\mathrm{HaH}$ completed the care programme at home, whereas $11.5 \%$ of $\mathrm{AH}$ patients continued their care in a long-term facility after hospital discharge, with an average daily cost of US $\$ 174.7$ for a mean period of $25 \pm 8.7$ days. Overall, on a cost per patient per day basis, $\mathrm{HaH}$ care was less expensive than that given to the AH group (US\$101.4 461.3 vs $\mathrm{US} \$ 151.7 \pm 96.4$, $\mathrm{p}=0.002$ ). This RCT reflected the results of the published systematic review. $^{36}$

\section{Pulmonary embolism}

Our review identified one published nRCT of HaH (Rodriguez-Cerillo et al) for patients with pulmonary embolism, which was also included in a recent systematic review with seven other observational studies (Vinson et al). ${ }^{25} 37$ The high-quality review concluded that the overall incidence of mortality at 90 days was very low.

The nRCT compared HaH $(n=30)$ with $\mathrm{AH}(\mathrm{n}=31)$ and was at high risk of bias overall. ${ }^{25}$ No distinctions between primary and secondary outcomes were made. Mean length of stay was not statistically different comparing HaH with the AH group (8.9 days (7-14 days) vs 10.6 days (6-20 days) ). No patients treated at home required unexpected return to hospital during admission. There was no major bleeding, thrombosis or death in either group at 90 days in the nRCT. ${ }^{25}$ There were no cost data reported.

\section{Pneumonia}

Our review identified one RCT (Carratala et al) published and included in a recent systematic review (Chalmers et al) that also described a further five studies comprising a variety of designs. ${ }^{2638}$ The RCT compared HAH $(n=110)$ with $\mathrm{AH}(\mathrm{n}=114)$ and was at low risk of bias. The primary outcome was the percentage of patients with an 'overall successful outcome' according to seven predefined criteria, ${ }^{26}$ while secondary outcomes were patients' QoL and satisfaction.

An overall successful outcome was achieved in $83.6 \%$ of $\mathrm{HaH}$ patients and $80.7 \%$ of $\mathrm{AH}$ patients (absolute difference $2.9 \%$ (95\% CI 7.1 to 12.9$)$ ). Subsequent hospital admissions were comparable between groups $(6.3 \%$ vs $7.0 \%)$. More $\mathrm{HaH}$ patients were satisfied with their overall care $(91.2 \%$ vs $79.1 \%$; ab $12.1 \%$ (CI $1.8 \%$ to $22.5 \%$ )). Reported QoL scores were comparable between groups as was the percentage of patients with adverse drug reactions $(9.1 \%$ vs $9.6 \%)$, medical complications $(0.9 \%$ vs $2.6 \%)$ and overall mortality $(0.9 \%$ vs $0 \%)$ for HAH and AH patient groups, respectively. There were no cost data presented. This RCT data reflect the result of the systematic review by Chalmers $e a l^{38}$

\section{Stroke}

One RCT on HaH for stroke patients (Kalra et al) was published and also included in two previous systematic reviews. ${ }^{27} 3539$ This RCT was at low risk of bias. The primary outcome measure was death or institutionalisation at 1 year. This three-arm study randomised patients 
into care on a stroke unit (SU) (n=152), care in a general ward $(\mathrm{GW})$ with stroke expert advice $(\mathrm{n}=152)$ and $\mathrm{HaH}$ with stroke expert advice $(n=153)$ within 72 hours after recruitment in the ED department.

Mortality and institutionalisation at 1 year were lower in the SU group compared with either the GW (14\% vs. $30 \%$, $\mathrm{p}<0.001)$ or $\mathrm{HaH}$ groups $(14 \%$ vs $24 \%, \mathrm{p}=0.03)$. Significantly fewer patients cared for on the SU died compared with those in the GW group ( $9 \%$ vs $23 \%, \mathrm{p}=0.001$ ). The SU group showed greater improvement on basic activities of daily living compared with the other two groups (change in Barthel Index: 10 vs 7, $\mathrm{p}<0.002$ ). QoL at three months was significantly better in $\mathrm{SU}$ and $\mathrm{HaH}$ patients. There was greater dissatisfaction with care in the GW group compared with $\mathrm{SU}$ or $\mathrm{HaH}$ groups. The total costs of stroke care per patient over 12 months (data collected in 2005-2008) were £11450 for the SU group, £9527 for the GW group and $£ 6840$ for the HaH group.

\section{Uncomplicated diverticulitis}

Our systematic review found one nRCT (Rodriguez-Cerrillo et al).$^{28}$ This study was also included in a recent, moderate-quality integrative review on admission-avoidance $\mathrm{HaH}$ services. ${ }^{40}$ This nRCT compared $\mathrm{HaH}(\mathrm{n}=34)$ with $\mathrm{AH}(\mathrm{n}=18)$ for patients with uncomplicated diverticulitis and was, overall, at high risk of bias with no defined primary or secondary outcomes. No statistical detail was provided about any of the data presented. None of the patients treated at home were transferred to AH. The mean length of stay in the intervention group was 9 days, compared with 10 days in $\mathrm{AH}$. $\mathrm{HaH}$ treatment was associated with a cost reduction of $€ 1368$ per patient.

\section{Older population with acute medical problems}

There were three studies identified published over five papers $^{2-33}$ and no relevant recent systematic reviews. One nRCT recruited acutely ill older persons and was published across three separate papers (Leff et al, main publication $).{ }^{29-31}$ This nRCT compared $\mathrm{HaH}(\mathrm{n}=169)$ with AH $(n=286)$ with the majority of patients being identified the morning after admission. The study was at high risk of bias. ${ }^{29}$ There was no distinction made between primary and secondary outcomes. Patients treated with $\mathrm{HaH}$ had a shorter length of stay compared with those given $\mathrm{AH}$ care (3.2 vs 4.9 days, $\mathrm{p}=0.004)$. The mean treatment cost was lower for $\mathrm{HaH}$ care than for AH care (US\$5081 vs US $\$ 7480, p<0.001)$. Eight weeks after admission, there were no differences in the use of health services between $\mathrm{HaH}$ and $\mathrm{AH}$ patients in terms of ED visits $(0.23$ (SD $0.66), 0.22$ (SD 0.57)) or readmission (0.28 (SD 0.59), 0.27 (SD 0.55)).

The nRCT by Crilly et al recruited elderly nursing home patients presenting at ED but who were willing to receive care back in their nursing home $(n=62)$ and compared these with historical control care home patients who had been hospitalised $(n=115)$. The study was at high risk of bias, ${ }^{32}$ and no primary outcomes were specified. Intervention participants experienced a longer time in ED than those who had been admitted into hospital (9.94 vs 7.01 hours, $\mathrm{p}=0.005$ ) but required less time being subsequently cared for (2.19 vs 6.2 days, $\mathrm{p}<0.001)$. Overall, the length of an episode of care in days (9.56 (1.26) vs. $6.20(0.59)$ days, $\mathrm{p}=0.14$ ) and the number of readmissions within 28 days ( 11.3 vs $11.3, \mathrm{p}=0.99$ ) were not statistically different between the two groups. There were no mortality or cost data presented.

The nRCT by Lau et al assessed residents of a care home presenting at ED who were subsequently treated back in their care home $(\mathrm{n}=95)$ and compared data with historical hospital controls (ie, not from care homes) $(n=167){ }^{33}$ No primary outcomes were specified, and the study was at high risk of bias. Length of stay was significantly shorter for those in the intervention group compared with the controls (2 vs 11 days, $\mathrm{p}<0.001$ ), although mortality (11 $(11.6 \%)$ vs $20(12.0 \%), \mathrm{p}=0.924)$ and readmission rates (39 $(41.1 \%)$ vs $68(40.7 \%), \mathrm{p}=0.963)$ at 6 months were comparable between groups. There were no cost data presented.

\section{Characteristics of those older patients for whom the decision} to admit to hospital may be unclear

Fifteen of the studies included in our systematic review recruited a population with a mean age of more than 75 years, despite the inclusion criterion specifying those over 65 years. While 9 out of 19 studies specifically stated that their recruited population was multi-morbid, it is plausible that all the study populations were and so this is very likely to be a factor that impacts on decision making in acute medical care. Eight studies specified a particular degree of severity for dementia as an inclusion criterion, but in practice, this is a difficult assessment to make in the acute care context. There were inclusion/exclusion criteria in nine of the studies that specified the importance of taking account of an individual's home situation, social support networks and coping abilities as part of the decision-making process (online supplementary file 7).

\section{DISCUSSION}

\section{Summary of principal findings}

The findings of our systematic review show that alternatives to AH care at the point of potential admission for people aged over 65 years can be safe, with comparable mortality and clinical outcomes across a range of acute and chronic conditions. They also have the potential to reduce healthcare spending. The exception to the evidence of benefit of $\mathrm{HaH}$ is the treatment of patients with stroke, who fare much worse with $\mathrm{HaH}$ intervention compared with treatment in an SU. The authors of this study suggest that these differences are due to the overall expertise available in $\mathrm{SU}$ as opposed to care given by generic hospital or homecare staff advised by specialised stroke health professionals. It is recommended therefore that, in most cases, in line with current NHS practice for stroke, care should to be provided in specialist units. ${ }^{41}$ The key features of older patients for whom the decision to admit may be uncertain are age more than 75 years, 
comorbidities/multi-morbidities, dementia, home situation, social support and individual coping abilities.

\section{Comparison with previous literature}

As part of our systematic review, any relevant systematic review published in 2010-2016 was included and referred to when discussing the more recent studies. All of these reviews were on the topic of $\mathrm{HaH}$ interventions. In addition to being older evidence, some of the previous reviews in contrast to our own included a number of uncontrolled observational studies. Some also included studies in which $\mathrm{HaH}$ interventions were applied in the non-emergency or postdischarge settings. By contrast, our systematic review focuses on bringing together controlled studies on alternatives to acute hospitalisation at the point of potential admission for those over 65 years old.

\section{Clinical and research implications}

For health professionals, making a decision to admit an older patient can prove very difficult. Decision making for each individual patient draws upon a range of professional experience and expertise and should also be influenced by broader factors such as living conditions and individual/family/carer coping, in addition to care preferences. If alternatives to acute admission are available, health professionals must be confident about using these alternative pathways for their patients, ${ }^{5}$ and while many of the interventions in this review may provide viable alternatives to acute care, they may not exist in some healthcare communities or geographical regions. Nevertheless, our review suggests that, where established alternatives to admission exist, clinicians should offer these with a degree of confidence and not assume that hospital admission is always the best or safest option for their patient.

Future research should aim to provide more comprehensive evidence of both the clinical and cost-effectiveness of a wider range of hospital alternatives for a greater range of health issues, as well as exploring in more detail the determinants and outcomes of decision making under conditions of uncertainty. Many of the studies included in this review recruited highly defined populations, and it would be helpful to understand whether the findings can be replicated in more general patient groups. There is also much to be done to improve the collection of data on patient-related outcomes, carer and health professional acceptability and costs.

\section{Strengths and limitations of review}

Our systematic review was conducted to high methodological standards. ${ }^{42}$ The majority of evidence presented is based on $\mathrm{HaH}$ services, although this includes treatment of a wide range of conditions. While not all the included studies were randomised or considered to be at low risk of bias, these issues are clearly highlighted and the included studies cover a variety of alternative approaches to hospital admission. The majority of the included studies offer little or no cost data, which makes it difficult to assess the cost-effectiveness of any these alternatives to AH care.
While writing our protocol, we planned to carry out a meta-analysis on suitable data. However, the data we identified were insufficient, in terms of quantity (ie, often drawn from a single study), quality (ie, from nRCT) or homogeneity. Where sufficient data were identified-on $\mathrm{HaH}$ for $\mathrm{HF}$ - an analysis had already been conducted within a previous review. ${ }^{34}$

In conclusion, this systematic review describes and assesses evidence on alternatives to acute care for older patients and shows that many of the options available are safe and appear to reduce resource use.

\section{Author affiliations}

${ }^{1}$ Centre for Academic Primary Care, School of Social and Community Medicine, University of Bristol, Bristol, UK

${ }^{2}$ Brunelcare, Saffron Gardens, Bristol, UK

${ }^{3}$ Health Economics at Bristol, School of Social and Community Medicine University of Bristol, Bristol, UK

${ }^{4}$ Bristol Randomised Trials Collaboration, University of Bristol, Bristol, UK ${ }^{5}$ Faculty of Health and Applied Sciences, University of the West of England, Bristol, UK ${ }^{6}$ Department of Emergency Medicine, University Hospitals Bristol NHS Foundation Trust, Bristol, UK

Acknowledgements We thank Ben Davies of the Centre for Academic Primary Care, School of Social and Community Medicine at the University of Bristol for providing systematic review support to AH; Helen England, formerly of Bris-DOC, for providing advice to SP and the wider study team; and Sue Jenkins, lay representative, who co-led a patient and public involvement session with MC exploring the relevance of the study findings with a group of older lay representatives including Alma Brooks, Matt Pepper, Joan Pepper, Tom Doyle and Liz Banks-Jones.

Contributors ALH: lead systematic reviewer conducting all stages of the review and was responsible for the initial draft of paper. MC: protocol of systematic review is based on outline from National Institute for Health Research Programme Development Grant in which MC had a significant role; specific expertise in patient and public involvement; contributing to discussion as the review progressed; commenting and editing on the drafts of the paper. AH: specific expertise in patient-related outcomes; contributing to discussion as the review progressed; commenting and editing on the drafts of the paper. WH: specific expertise in health economics; contributing to discussion as the review progressed; commenting and editing on the drafts of the paper. CM: specific expertise in trial design and statistical analysis; contributing to discussion as the review progressed; commenting and editing on the drafts of the paper. JB: professor of emergency care; contributing to discussion as the review progressed; commenting and editing on the drafts of the paper. SP: principal investigator; professor of primary health care; third reviewer of data; commenting and editing on the drafts of the paper.

Funding This paper presents research funded by the National Institute for Health Research (NIHR) as part of their Programme Development Grant scheme (RP-DG1213-10004)

Disclaimer The views expressed are those of the authors and not necessarily those of the National Health Service, NIHR or the Department of Health.

Competing interests None declared.

Provenance and peer review Not commissioned; externally peer reviewed.

Data sharing statement This is a systematic review, and all the data we have collected are either in the main text and table or in the appendices and therefore freely available.

Open Access This is an Open Access article distributed in accordance with the Creative Commons Attribution Non Commercial (CC BY-NC 4.0) license, which permits others to distribute, remix, adapt, build upon this work non-commercially, and license their derivative works on different terms, provided the original work is properly cited and the use is non-commercial. See: http://creativecommons.org/licenses/by-nc/4.0/

(c) Article author(s) (or their employer(s) unless otherwise stated in the text of the article) 2017. All rights reserved. No commercial use is permitted unless otherwise expressly granted. 


\section{REFERENCES}

1. D'Souza S, Guptha S. Preventing admission of older people to hospital. BMJ 2013;346:f3186.

2. Philp I. Community services. reasons for redesigning care for older people. Health Serv J 2012;122:30-2.

3. NHS England. Bed availability and occupancy, 2013. https://www. england.nhs.uk/statistics/statistical-work-areas/bed-availability-andoccupancy/. (accessed 20 Dec 2016).

4. Hammond CL, Pinnington LL, Phillips MF, et al. A qualitative examination of inappropriate hospital admissions and lengths of stay. BMC Health Serv Res 2009;9:44.

5. Walsh B, Lattimer V, Wintrup J, et al. Professional perspectives on systemic barriers to admission avoidance: learning from a system dynamics study of older people's admission pathways. Int J Older People Nurs 2015;10:105-14.

6. The King's Fund,https://www.kingsfund.org.uk/ accessed 18 Jan 2017

7. AHRQ,http://www.ahrq.gov/ accessed 18 Jan 2017

8. EPOC risk of bias criteria http://epoc.cochrane.org/sites/epoc. cochrane.org/files/public/uploads/Suggested\%20risk\%20of\% 20bias\%20criteria\%20for\%20EPOC\%20reviews.pdf accessed 18 Jan 17

9. Shea BJ, Grimshaw JM, Wells GA, et al. Development of AMSTAR: a measurement tool to assess the methodological quality of systematic reviews. BMC Med Res Methodol 2007;7:10

10. Mason S, Knowles E, Colwell B, et al. Effectiveness of paramedic practitioners in attending 999 calls from elderly people in the community: cluster randomised controlled trial. BMJ 2007;335:919.

11. Gray JT, Walker A. Avoiding admissions from the ambulance service: a review of elderly patients with falls and patients with breathing difficulties seen by emergency care practitioners in South Yorkshire. Emerg Med J 2008;25:168-71.

12. Mason S, O'Keeffe $\mathrm{C}$, Knowles $\mathrm{E}$, et al. A pragmatic quasiexperimental multi-site community intervention trial evaluating the impact of emergency care practitioners in different UK health settings on patient pathways (NEECaP trial). Emerg Med J 2012;29:47-53.

13. Sun BC, McCreath $\mathrm{H}$, Liang LJ, et al. Randomized clinical trial of an emergency department observation Syncope protocol versus routine inpatient admission. Ann Emerg Med 2014;64:167-75.

14. Benaiges D, Chillarón JJ, Carrera MJ, et al. Efficacy of treatment for hyperglycemic crisis in elderly diabetic patients in a day hospital. Clin Interv Aging 2014;9:843-9.

15. Salvi F, Morichi V, Grilli A, et al. A geriatric emergency service for acutely ill elderly patients: pattern of use and comparison with a conventional emergency department in Italy. J Am Geriatr Soc 2008;56:2131-8.

16. Vicente V, Svensson L, Wireklint Sundström B, et al. Randomized controlled trial of a prehospital decision system by emergency medical services to ensure optimal treatment for older adults in Sweden. J Am Geriatr Soc 2014;62:1281-7.

17. Garåsen $\mathrm{H}$, Windspoll $\mathrm{R}$, Johnsen $\mathrm{R}$, et al. Intermediate care at a community hospital as an alternative to prolonged general hospital care for elderly patients: a randomised controlled trial. BMC Public Health 2007:7:68. Main publication.

18. Garåsen $\mathrm{H}$, MagnussenJ,WindspollR, et al. Elderly patients in hospitals or nursing homes in intermediæravdeling-A cost analysis. Tidsskr Nor Legeforen 2008a;128:283-5. (Danish).

19. Garåsen $\mathrm{H}$, Windspoll $\mathrm{R}$, Johnsen $\mathrm{R}$. Long-term patients' outcomes after intermediate care at a community hospital for elderly patients: 12-month follow-up of a randomized controlled trial. Scand J Public Health 2008;36:197-204.

20. Mendoza H, Martín MJ, García A, et al. 'Hospital at home' care model as an effective alternative in the management of decompensated chronic heart failure. Eur J Heart Fail 2009;11:1208-13. main publication.

21. García-Soleto A, Parraza-Diez N, Aizpuru-Barandiaran F, et al. Comparative study of quality of life after hospital-at-home or in- patient admission for acute decompensation of chronic heart failure. World J Cardiovasc Dis 2013;03:174-81.

22. Tibaldi V, Isaia G, Scarafiotti C, et al. Hospital at home for elderly patients with acute decompensation of chronic heart failure: a prospective randomized controlled trial. Arch Intern Med 2009;169:1569-75.

23. Patel H, Shafazand M, Ekman I, et al. Home care as an option in worsening chronic heart failure -- a pilot study to evaluate feasibility, quality adjusted life years and cost-effectiveness. Eur J Heart Fail 2008;10:675-81.

24. Aimonino Ricauda N, Tibaldi V, Leff B, et al. Substitutive "hospital at home" versus inpatient care for elderly patients with exacerbations of chronic obstructive pulmonary disease: a prospective randomized, controlled trial. J Am Geriatr Soc 2008;56:493-500.

25. Rodríguez-Cerrillo M, Alvarez-Arcaya A, Fernández-Díaz E, et al. A prospective study of the management of non-massive pulmonary embolism in the home. Eur J Intern Med 2009;20:598-600.

26. Carratalà J, Fernández-Sabé N, Ortega L, et al. Outpatient care compared with hospitalization for community-acquired pneumonia: a randomized trial in low-risk patients. Ann Intern Med 2005;142:165-72.

27. Kalra L, Evans A, Perez I, et al. A randomised controlled comparison of alternative strategies in stroke care. Health Technol Assess 2005;9:1-79. iii-iv.

28. Rodríguez-Cerrillo M, Poza-Montoro A, Fernandez-Diaz E, et al. Treatment of elderly patients with uncomplicated diverticulitis, even with comorbidity, at home. Eur J Intern Med 2013;24:430-2.

29. Leff B, Burton L, Mader SL, et al. Hospital at home: feasibility and outcomes of a program to provide hospital-level care at home for acutely ill older patients. Ann Intern Med 2005;143:798-808. Main publication.

30. Leff B, Burton L, Mader SL, et al. Comparison of functional outcomes associated with hospital at home care and traditional acute hospital care. J Am Geriatr Soc 2009;57:273-8.

31. Frick KD, Burton LC, Clark R, et al. Substitutive hospital at home for older persons: effects on costs. Am J Manag Care 2009;15:49-56.

32. Crilly J, Chaboyer W, Wallis M, et al. An outcomes evaluation of an Australian hospital in the Nursing Home admission avoidance programme. J Clin Nurs 2011;20:1178-87.

33. Lau L, Chong CP, Lim WK. Hospital treatment in residential care facilities is a viable alternative to hospital admission for selected patients. Geriatr Gerontol Int 2013;13:378-83.

34. Qaddoura A, Yazdan-Ashoori P, Kabali C, et al. Efficacy of hospital at home in patients with Heart failure: a systematic review and MetaAnalysis. PLoS One 2015;10:e0129282.

35. Shepperd S, lliffe S, Doll HA, et al. Admission avoidance hospital at home. Cochrane Database Syst Rev 2016;9:CD007491.

36. Jeppesen E, Brurberg KG, Vist GE, et al. Hospital at home for acute exacerbations of chronic obstructive pulmonary disease. Cochrane Database Syst Rev 2012;5:CD003573.

37. Vinson DR, Zehtabchi S, Yealy DM. Can selected patients with newly diagnosed pulmonary embolism be safely treated without hospitalization? A systematic review. Ann Emerg Med 2012;60:651-62.

38. Chalmers JD, Akram AR, Hill AT. Increasing outpatient treatment of mild community-acquired pneumonia: systematic review and metaanalysis. Eur Respir J 2011;37:858-64.

39. Caplan GA. A meta-analysis of "hospital in the home". Med J Aust 2013;198:195-6.

40. Varney J, Weiland TJ, Jelinek G. Efficacy of hospital in the home services providing care for patients admitted from emergency departments. Int J Evid Based Healthc 2014;12:128-41.

41. NICE pathway for stroke https://pathways.nice.org.uk/pathways/ stroke accessed 20 dec 2016

42. Higgins JPT, Green S, eds. Cochrane Handbook for Systematic Reviews of Interventions Version 5.1.0: The Cochrane Collaboration, 2011. (accessed Mar 2011). 